\title{
A BRB Based Fault Prediction Method of Complex Electromechanical Systems
}

\author{
Bangcheng Zhang, ${ }^{1}$ Xiaojing Yin, ${ }^{1}$ Zhanli Wang, \\ Xiaoxia Han, ${ }^{2}$ and Zhi Gao ${ }^{1}$ \\ ${ }^{1}$ School of Mechatronic Engineering, Changchun University of Technology, Changchun 130012, China \\ ${ }^{2}$ High-Tech Institute of Xian, Xi'an 710025, China \\ Correspondence should be addressed to Zhanli Wang; wangzl@mail.ccut.edu.cn
}

Received 15 September 2014; Accepted 14 December 2014

Academic Editor: Gang Li

Copyright (C) 2015 Bangcheng Zhang et al. This is an open access article distributed under the Creative Commons Attribution License, which permits unrestricted use, distribution, and reproduction in any medium, provided the original work is properly cited.

Fault prediction is an effective and important approach to improve the reliability and reduce the risk of accidents for complex electromechanical systems. In order to use the quantitative information and qualitative knowledge efficiently to predict the fault, a new model is proposed on the basis of belief rule base (BRB). Moreover, an evidential reasoning (ER) based optimal algorithm is developed to train the fault prediction model. The screw failure in computer numerical control (CNC) milling machine servo system is taken as an example and the fault prediction results show that the proposed method can predict the behavior of the system accurately with combining qualitative knowledge and some quantitative information.

\section{Introduction}

Modern complex electromechanical systems are usually composed of the multiphysical processes such as machinery, electricity, and hydromantic [1]. A long period is needed to design and manufacture a complex electromechanical system. Moreover, the cost for maintenance of these systems is high. Therefore, it is important to predict the different kinds of faults of the complex electromechanical systems [2].

A complex electromechanical system has the following characteristics. Firstly, it is composed of some subsystems. Because of the coupling, the behaviors of the system cannot be determined independently by analyzing the behaviors of each part. Secondly, it is difficult to establish the analytical model. Thirdly, the fault processes of a complex electromechanical system may be reflected by some states or parameters at the same time. Finally, the complex electromechanical system is a dynamic system. Thus, if the fault of a complex electromechanical system is predicted, some characteristic variables should be chosen and the fault prediction algorithm should be online which can ensure that the fault can be predicted in real-time.
The current fault prediction methods can be divided into the method based on quantitative information, the method based on qualitative information, and the method based on hybrid information. The quantitative information based method includes Kalman filter, grey theory [3], neural network [4-6], time series [7], and data fusion based methods. This kind of methods needs the analytical models of the systems. In this method, the structures of the systems and the mechanism information are used. But it is difficult to analyze and interpret the forecasting results. The qualitative knowledge based method [8] includes the expert system and time sequence logic based methods. But the combination explosion may happen and the forecasting result is not accurate. The hybrid information based method such as fuzzy mathematics, neural network, Kalman filter, and wavelet plays the advantages of various kinds of a single method effectively. However, most existing methods are two kinds of fusion method based on quantitative information without qualitative knowledge application, which may restrict the use of various types of knowledge comprehensively to improve the accuracy of the fault prediction. The belief rule base (BRB) could make full use of the prior knowledge of expert and 


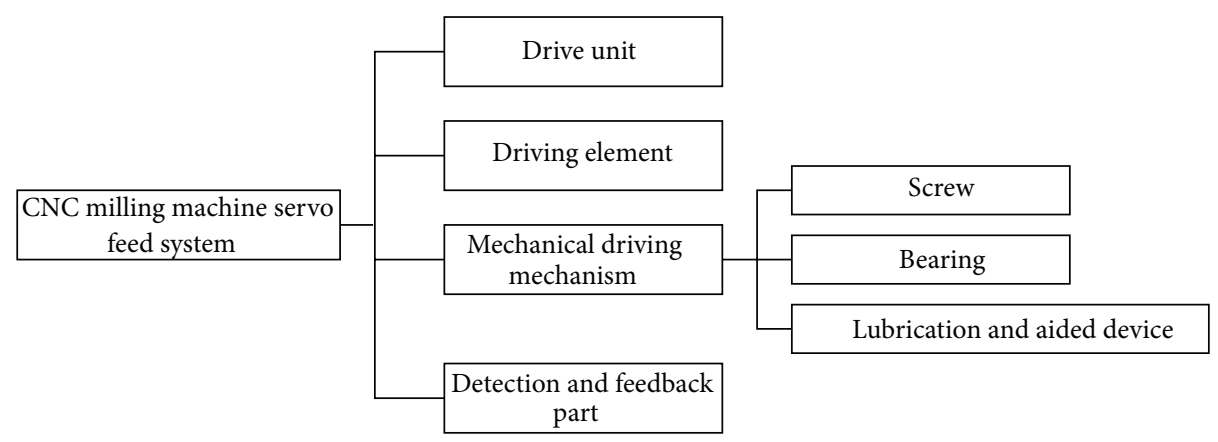

FIgURE 1: Servo feed system of CNC milling machine.

quantitative information. Moreover the evidential reasoning (ER) is used to update the characteristics online $[9,10]$. These guaranteed the accurate prediction of the fault [11-13].

In this paper, BRB is used to establish the fault prediction model of a complex electromechanical system. BRB overcomes the shortcomings of a single method based on quantitative or qualitative knowledge $[14,15]$. This method realizes the layered and comprehensive fault prediction of a complex electromechanical system through combining BRB with the model of a complex electromechanical system. Taking the CNC machine tool that is a typical complex electromechanical system as an example [16], this paper describes the detailed process of layering and comprehensive fault prediction about the servo system. The results show that this method can predict the fault behaviors of a complex electromechanical system accurately and may be applied in the engineering practice.

\section{The System Modeling Based on the Characteristics}

A complex electromechanical system is composed of some subsystems with strong coupling, time-varying, and strong nonlinear characteristics. As temperature, blood pressure, and other indicators can reflect the health of the human body system, there are also some characteristics that can reflect the running condition of a complex electromechanical system [17]. According to these characteristics, the fault prediction model of a complex electromechanical system can be built.

Firstly, it is assumed that a complex electromechanical system is composed of $n$ parts, and the running condition of $j$ th part can be represented by the available feature $\mathbf{N}_{j}$, which is a multiparameter and where $j=1,2, \ldots, n$. The running condition of the entire electromechanical system can be described by the following model:

$$
y=g\left(\mathbf{N}_{1}, \mathbf{N}_{2}, \ldots, \mathbf{N}_{n}\right)
$$

where $y$ denotes the running condition of the whole complex electromechanical system. $g$ denotes a nonlinear mapping.

In addition, the running condition of each component $\mathbf{N}_{j}$ can be represented by some characteristic variables and it is assumed that the $j$ th component can be represented by the $M$ characteristic variables, recorded as $h_{1}, h_{2}, \ldots, h_{M}$ :

$$
f=l\left(h_{1}, h_{2}, \ldots, h_{M}\right)
$$

where $f$ denotes the running condition of $j$ th component. $l$ denotes a nonlinear mapping.

Then the following model that can denote the running condition of a complex electromechanical system can be obtained by putting (2) into (1):

$$
y=g(f) .
$$

\section{Modeling the CNC Machine Tool Servo System}

3.1. Composition of CNC Machine Tool Servo System. In order to establish the fault prediction model of the CNC milling machine servo feed system, its structure should be analyzed firstly. As shown in Figure 1, the CNC milling machine servo feed system is mainly composed of the drive unit, driving element, mechanical driving mechanism, actuator, detection, and feedback part.

3.2. Fault Mechanism Analysis of the CNC Milling Machine Servo Feed System. The fault of the driving mechanism mainly includes that the motor drive power cannot be passed to the executive element. This kind of fault often occurs in machine tool guide, coupling screw, bearing parts, and so on. Overload and other problems often occurred in these parts when they have faults such as clearance being too large, vibration, or wear. In drive system, servo motor is the main part where malfunction happens easier. Faults of drive system mainly include drive control unit fault and servo motor fault. Detection component often become large errors of feedback data or no feedback $[18,19]$.

In servo feed transmission, bearing and screw feed transmission mechanism are the most prone to failure, so the fault mechanism of bearing and lead screw nut pair parts are analyzed. Under normal working conditions, too large load or poor lubrication can lead to different forms of damage which would cause machine tool state degradation [20]. The types of rolling bearing fault mainly include surface knit crack, surface spelling, and bearing burning. Typical failure damage 


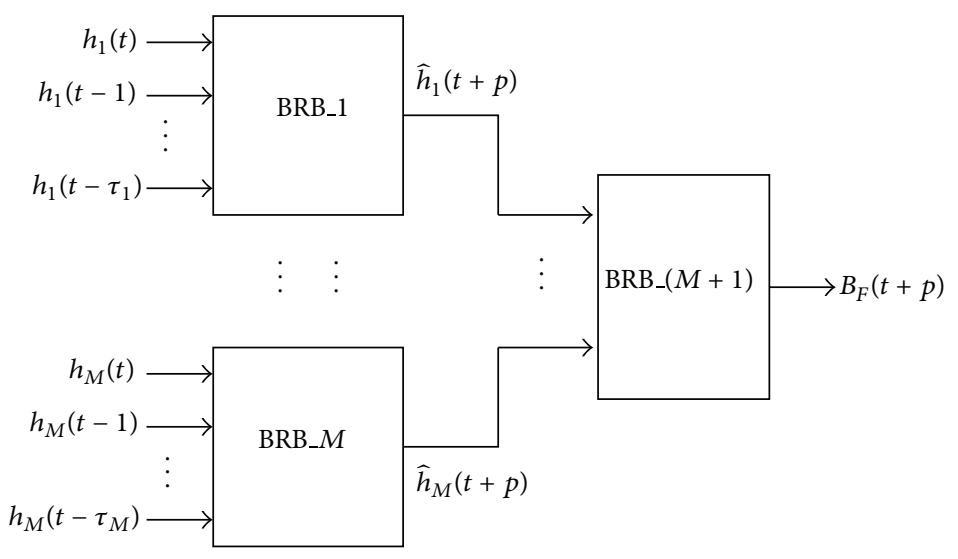

FIGURE 2: BRB fault prediction model.

of ball screw drive system consists of surface damage, serious deformation, and failure. The surface damage mainly includes removing fatigue failure, wear, and corrosion failure.

The motor fault is one of the main faults in the drive system. Finding the motor fault timely and accurately can avoid economic loss and serious safety accidents. According to the main part of motor, the fault can be divided into the failure of the stator, the rotor fault, and bearing failure.

Detection device plays an important role in the whole control system, and its performance directly determines the precision of the detection system, which affects the machining accuracy of CNC machine tools. Encoder failure is a typical failure in detection device. Encoder as a detecting element of closed-loop system or semi-closed loop system, its feedback signal directly affects the adjustment of the motor speed. Generally, the faults are divided into the encoder to throw code and complete failure.

3.3. The Model of CNC Milling Machine Servo Feed System. According to the structure and the fault mechanism analysis of the CNC milling machine servo feed system, the model of CNC milling machine servo feed system is built as follows, referencing model of complex electromechanical system.

$\mathrm{CNC}$ milling machine servo feed system is composed of four parts; the model of CNC machine tool servo system is built as follows by (1):

$$
y=g\left(\mathbf{N}_{1}, \mathbf{N}_{2}, \mathbf{N}_{3}, \mathbf{N}_{4}\right) .
$$

In (4), $y$ denotes the running condition of CNC machine tool servo system. $g$ denotes the nonlinearity mapping.

By the same token, the model of mechanical transmission system is as follows:

$$
y^{\prime}=g^{\prime}\left(\mathbf{N}_{1}, \mathbf{N}_{2}, \mathbf{N}_{3}\right) .
$$

In (5), $y^{\prime}$ is the running condition of CNC machine tool servo system. $g^{\prime}$ denotes the nonlinearity mapping.

\section{A BRB Based Fault Prediction Model}

On the basis of analyzing the fault mechanism, a series of available features are chosen to reflect the running condition of each part, so, $h_{m}$ is confirmed. Then, the belief rule base (BRB) is used to establish the fault prediction [11]. A belief rule in $\mathrm{BRB}$ is described as follows:

$$
\begin{gathered}
R_{k}: \text { If } h_{1} \text { is } H_{1}^{k} \wedge h_{2} \text { is } H_{2}^{k} \cdots \wedge h_{M} \text { is } H_{M}^{k} \text {, } \\
\text { Then }\left\{\left(D_{1}, \beta_{1, k}\right), \ldots,\left(D_{N}, \beta_{N, k}\right)\right\}
\end{gathered}
$$

with a rule weight $\theta_{k}$ and attribute

$$
\text { weight } \delta_{1, k}, \delta_{2, k}, \ldots, \delta_{M_{k}, k} \text {, }
$$

where $R_{k}$ denotes the $k$ th rule belief rule. $\mathbf{h}=\left[h_{1}, h_{2}, \ldots, h_{M}\right]$ is antecedent attributes in the $k$ th rule, and $h_{M}$ is an element of $N_{i}$ in (4), same as $a_{i}$ in (1). $\mathbf{H}^{k}=\left\{H_{1}^{k}, H_{2}^{k}, \ldots, H_{M}^{k}\right\}$ is a collection of input reference values in the $k$ th rule. $D=\left[D_{1}, D_{2}, \ldots, D_{N}\right]$ is a set of referential values for the antecedent attribute. $\beta^{k}=\left[\beta_{1, k}, \beta_{2, k}, \ldots, \beta_{N, k}\right]$ is a set of belief degrees assessed to $D$.

Figure 2 shows a BRB based fault prediction model [21], where $p$ denotes the forecasting step and $B$ denotes the preset threshold. $\widehat{h}_{m}(t+p)(m=1, \ldots, M)$ denotes the estimates in $(t+p)$ moment of $h_{m}$. In Figure 2, the first $M$ BRBs, that is, $\mathrm{BRB}_{-} 1, \ldots, \mathrm{BRB} \_M$, which are given in (6) belong to the traditional rules in which the consequents are either $100 \%$ normal or $100 \%$ failure. When the system is in a defective but still operational stage, such rules which are abovementioned have a limited capacity. In order to solve this problem, $\mathrm{BRB}_{-}(M+1)$ is used to determine the system running condition by extending the (6) [21]. Consider

$$
\begin{aligned}
& R_{1}: \text { If } h_{1} \leq h_{1}^{d} \wedge \cdots \wedge h_{M} \leq h_{M}^{d}, \\
& \quad \text { Then }\left\{\left(D_{1}, 0\right),\left(D_{2}, 1\right)\right\}, \\
& \text { with a rule weight } \theta_{1} \\
& \quad \text { and attribute weight } \delta_{1,1}, \ldots, \delta_{M, 1}
\end{aligned}
$$




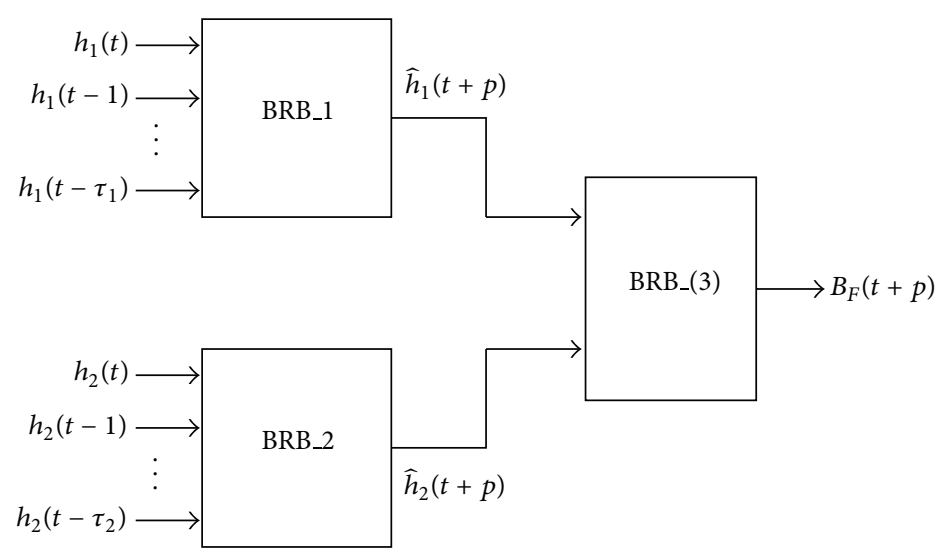

FIGURE 3: Prediction model of ball screw based on BRB.

$$
\begin{aligned}
& R_{G}: \text { If } h_{1} \in\left[h_{1}^{d n}, h_{1}^{u n}\right] \wedge \cdots \wedge\left[h_{M}^{d n}, h_{M}^{u n}\right], \\
& \text { Then }\left\{\left(D_{1}, 0\right),\left(D_{2}, 0\right)\right\} \\
& \text { with a rule weight } \theta_{G} \\
& \text { and attribute weight } \delta_{1, G}, \ldots, \delta_{M, G} \\
& \quad \vdots \\
& R_{3^{M}}: \text { If } h_{1} \geq h_{1}^{u} \wedge \cdots \wedge h_{M} \geq h_{M}^{u}, \\
& \text { Then }\left\{\left(D_{1}, 0\right),\left(D_{2}, 1\right)\right\},
\end{aligned}
$$

with a rule weight $\theta_{3^{M}}$ and attribute weight $\delta_{1,3^{M}}, \ldots, \delta_{M, 3^{M}}$,

where $\delta_{1,3^{M}}, \ldots, \delta_{M, 3^{M}}$ are the relative weights of the $M$ antecedent attributes used in the $k$ th rule. For each characteristic variable $h_{m}(m=1, \ldots, M)$, there are three referential values which include $h_{m}^{d}, h_{m}^{u}$, and $\left[h_{m}^{d n}, h_{m}^{u n}\right]$, so there are $3^{M}$ belief rules in $\mathrm{BRB}_{-}(M+1) . \theta_{k}\left(k=1, \ldots, 3^{M}\right)$ is the relative weight of the $k$ th rule. It is assumed that $\theta_{k}=1$ and $\delta_{m, k}=1$.

In practical engineering problems, the ER algorithm is used to update the parameters. The result of update is shown in $[21]$

$$
\begin{aligned}
& \mathbf{Q}_{m}(t+1) \\
& \quad=\prod_{E_{m}}\left\{\mathbf{Q}_{m}(t)+\frac{1}{t}\left[\Xi_{m}\left(\mathbf{Q}_{m}(t)\right)\right]^{-1} \Gamma_{m}\left(\mathbf{Q}_{m}(t)\right)\right\},
\end{aligned}
$$

where $\mathbf{Q}_{m}$ consists of the rule weights, attribute weights, and belief degrees satisfying the constraints. $E_{m}$ is a constraint set composed of the constraints. $\prod_{H_{m}}\{\cdot\}$ is the projection onto the constraint set $E_{m}$, ensuring that the estimation of $\mathbf{Q}_{m}$ can satisfy the given constraints.

$B_{F}(t+p)$ can be got through the model. If it is less than $B_{\mathrm{th}}$, the system can work normally. On the contrary, the system is in fault condition, where $B_{\text {th }}$ denotes a preset threshold.

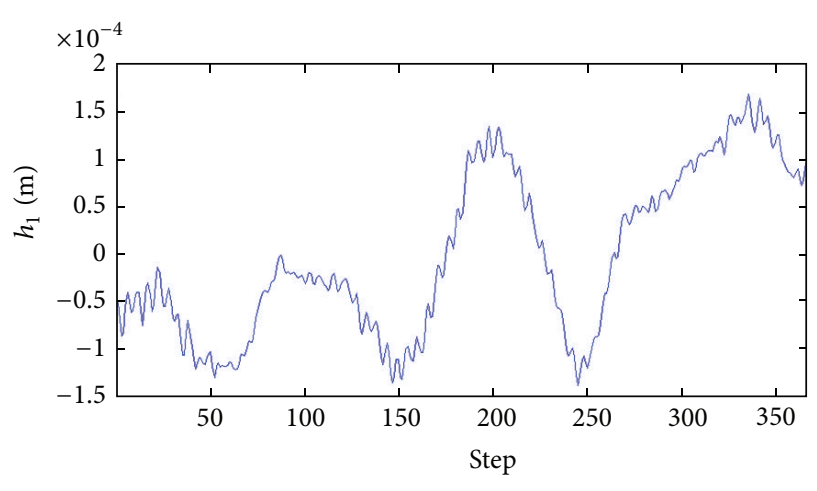

FIGURE 4: Test data of $h_{1}$.

\section{Fault Prediction of Screw in CNC Milling Machine Servo Feed System}

The running condition of the screw is one of the most characteristics which can represent the condition of the whole $\mathrm{CNC}$ system. In this section, the screw is chosen as an example to demonstrate the effectiveness of the proposed method.

Through the analysis of fault mechanism, two variables that are the vibration of screw and the surface roughness of the processing pieces are chosen to reflect the performance of screw. These two variables are denoted by $h_{1}$ and $h_{2}$, respectively.

The system model of screw can be got according to (4) as follows:

$$
f=g^{\prime \prime}\left(h_{1}, h_{2}\right),
$$

where $f$ denotes the running condition of screw. $h$ denotes a nonlinearity mapping.

So, the fault prediction model of screw is established as shown in Figure 3.

Figures 4 and 5 display the testing data of $h_{1}$ and $h_{2}$, respectively. The testing data are collected in the processing cast-iron at a low speed by the B\&K and laser interferometer, and the motor speed is $1000 \mathrm{r} / \mathrm{min}$ and the feed is $40 \mathrm{~mm} / \mathrm{min}$. The test rig is shown in Figure 6. 


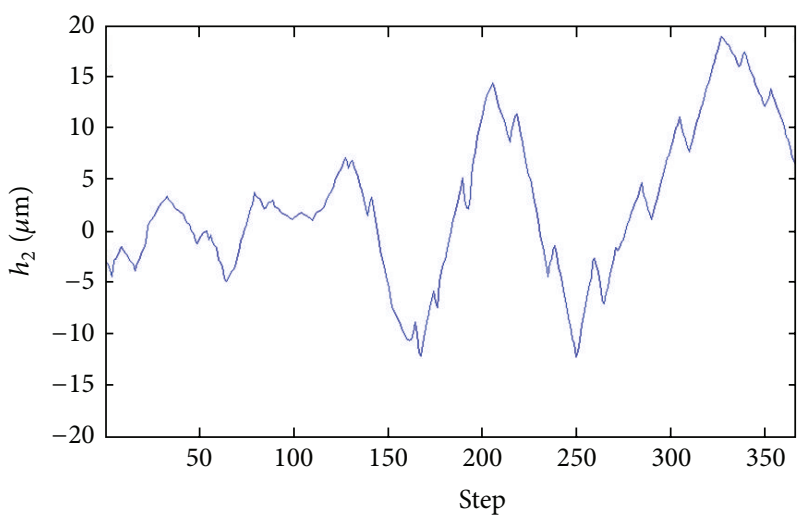

Figure 5: Test data of $h_{2}$.

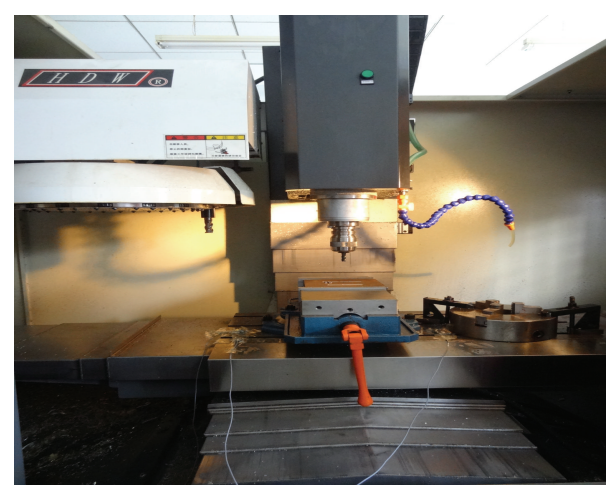

Figure 6: The test rig.

TABLE 1: The reference of $h_{1}(\mu \mathrm{m})$.

\begin{tabular}{lccc}
\hline Linguistic terms & S & M & L \\
\hline Numerical values & 0.5 & 1.5 & 2 \\
\hline
\end{tabular}

TABLE 2: The reference of $h_{2}(\mu \mathrm{m})$.

\begin{tabular}{llll}
\hline Linguistic terms & S & M & L \\
\hline Numerical values & 5 & 10 & 15 \\
\hline
\end{tabular}

In order to construct the BRB based forecasting models of the above two characteristics variables, the references of the characteristics variables are chosen at first. For both $h_{1}$ and $h_{2}$, three referential points are used and they are small $(S)$, medium $(M)$, and large $(L)$, which are given as follows:

$$
\begin{gathered}
H_{i}^{k_{1}} \in\{S, M, L\}, \quad H_{j}^{k_{2}} \in\{S, M, L\}, \\
i=1,2, \quad j=1,2, \\
\left\{D_{1}^{1}, D_{2}^{1}, D_{3}^{1}\right\} \in\{S, M, L\}, \\
\left\{D_{1}^{2}, D_{2}^{2}, D_{3}^{2}\right\} \in\{S, M, L\} .
\end{gathered}
$$

The above reference values are semantic which need to be quantified. The quantitative results of the two variables are listed in Tables 1 and 2.
TABLE 3: Initial belief degrees of BRB_1.

\begin{tabular}{lcc}
\hline Rule number & $\begin{array}{c}h_{1}(t-1) \text { and } \\
h_{1}(t-2)\end{array}$ & $h_{1}(t)$ distribution $\left\{D_{1}^{1}, D_{2}^{1}, D_{3}^{1}\right\}$ \\
\hline 1 & S and S & $\left\{\left(D_{1}^{1}, 0.65\right),\left(D_{2}^{1}, 0.25\right),\left(D_{3}^{1}, 0.1\right)\right\}$ \\
2 & S and M & $\left\{\left(D_{1}^{1}, 0.3\right),\left(D_{2}^{1}, 0.6\right),\left(D_{3}^{1}, 0.1\right)\right\}$ \\
3 & S and L & $\left\{\left(D_{1}^{1}, 0.9\right),\left(D_{2}^{1}, 0.05\right),\left(D_{3}^{1}, 0.05\right)\right\}$ \\
4 & M and S & $\left\{\left(D_{1}^{1}, 0.9\right),\left(D_{2}^{1}, 0.05\right),\left(D_{3}^{1}, 0.05\right)\right\}$ \\
5 & M and M & $\left\{\left(D_{1}^{1}, 0.2\right),\left(D_{2}^{1}, 0.6\right),\left(D_{3}^{1}, 0.2\right)\right\}$ \\
6 & M and L & $\left\{\left(D_{1}^{1}, 0\right),\left(D_{2}^{1}, 0.4\right),\left(D_{3}^{1}, 0.6\right)\right\}$ \\
7 & L and S & $\left\{\left(D_{1}^{1}, 1\right),\left(D_{2}^{1}, 0\right),\left(D_{3}^{1}, 0\right)\right\}$ \\
8 & L and M & $\left\{\left(D_{1}^{1}, 0.1\right),\left(D_{2}^{1}, 0.45\right),\left(D_{3}^{1}, 0.45\right)\right\}$ \\
9 & L and L & $\left\{\left(D_{1}^{1}, 0\right),\left(D_{2}^{1}, 0.1\right),\left(D_{3}^{1}, 0.9\right)\right\}$ \\
\hline
\end{tabular}

TABLE 4: Initial belief degrees of BRB_2.

\begin{tabular}{lcc}
\hline Rule number & $\begin{array}{c}h_{2}(t-1) \text { and } \\
h_{2}(t-2)\end{array}$ & $h_{2}(t)$ distribution $\left\{D_{1}^{2}, D_{2}^{2}, D_{3}^{2}\right\}$ \\
\hline 1 & S and S & $\left\{\left(D_{1}^{2}, 0.5\right),\left(D_{2}^{2}, 0.4\right),\left(D_{3}^{2}, 0.1\right)\right\}$ \\
2 & S and M & $\left\{\left(D_{1}^{2}, 0.3\right),\left(D_{2}^{2}, 0.5\right),\left(D_{3}^{2}, 0.2\right)\right\}$ \\
3 & S and L & $\left\{\left(D_{1}^{2}, 0.8\right),\left(D_{2}^{2}, 0.15\right),\left(D_{3}^{2}, 0.05\right)\right\}$ \\
4 & $\mathrm{M}$ and S & $\left\{\left(D_{1}^{2}, 0.45\right),\left(D_{2}^{2}, 0.45\right),\left(D_{3}^{2}, 0.1\right)\right\}$ \\
5 & $\mathrm{M}$ and M & $\left\{\left(D_{1}^{2}, 0.2\right),\left(D_{2}^{2}, 0.5\right),\left(D_{3}^{2}, 0.3\right)\right\}$ \\
6 & $\mathrm{M}$ and L & $\left\{\left(D_{1}^{2}, 0\right),\left(D_{2}^{2}, 0.4\right),\left(D_{3}^{2}, 0.6\right)\right\}$ \\
7 & $\mathrm{~L}$ and S & $\left\{\left(D_{1}^{2}, 1\right),\left(D_{2}^{2}, 0\right),\left(D_{3}^{2}, 0\right)\right\}$ \\
8 & $\mathrm{~L}$ and M & $\left\{\left(D_{1}^{2}, 0.1\right),\left(D_{2}^{2}, 0.45\right),\left(D_{3}^{2}, 0.45\right)\right\}$ \\
9 & $\mathrm{~L}$ and L & $\left\{\left(D_{1}^{2}, 0\right),\left(D_{2}^{2}, 0.1\right),\left(D_{3}^{2}, 0.9\right)\right\}$ \\
\hline
\end{tabular}

TABLE 5: Belief degrees of BRB_3.

\begin{tabular}{lll}
\hline Rule number & $h_{1}$ and $h_{2}$ & $\left\{D_{1}, D_{2}\right\}=\{N, F\}$ \\
\hline 1 & $h_{1}^{d}$ and $h_{2}^{d}$ & $\left\{\left(D_{1}, 0\right),\left(D_{2}, 1\right)\right\}$ \\
2 & $h_{1}^{d}$ and $h_{2}^{n}$ & $\left\{\left(D_{1}, 0\right),\left(D_{2}, 1\right)\right\}$ \\
3 & $h_{1}^{d}$ and $h_{2}^{u}$ & $\left\{\left(D_{1}, 0\right),\left(D_{2}, 1\right)\right\}$ \\
4 & $h_{1}^{n}$ and $h_{2}^{d}$ & $\left\{\left(D_{1}, 0\right),\left(D_{2}, 1\right)\right\}$ \\
5 & $h_{1}^{n}$ and $h_{2}^{n}$ & $\left\{\left(D_{1}, 1\right),\left(D_{2}, 0\right)\right\}$ \\
6 & $h_{1}^{n}$ and $h_{2}^{u}$ & $\left\{\left(D_{1}, 0\right),\left(D_{2}, 1\right)\right\}$ \\
7 & $h_{1}^{u}$ and $h_{2}^{d}$ & $\left\{\left(D_{1}, 0\right),\left(D_{2}, 1\right)\right\}$ \\
8 & $h_{1}^{u}$ and $h_{2}^{n}$ & $\left\{\left(D_{1}, 0\right),\left(D_{2}, 1\right)\right\}$ \\
9 & $h_{1}^{u}$ and $h_{2}^{u}$ & $\left\{\left(D_{1}, 0\right),\left(D_{2}, 1\right)\right\}$ \\
\hline
\end{tabular}

According to (6), the models of $h_{1}$ and $h_{2}$ are established. Then, experts give the initial belief degrees according to the historical information and the analysis of the running patterns of the screw, which are shown in Tables 3 and 4 . At the same time, the initial values of $\theta_{k_{1}}^{1}, \theta_{k_{2}}^{2}, \delta_{0, k_{1}}^{1}, \delta_{1, k_{1}}^{1}, \delta_{0, k_{2}}^{2}$, and $\delta_{1, k_{2}}^{2}$ are all set to 1 . In addition, $k_{1}=k_{2}=9$. So, the initial BRB_1 and BRB_2 are constructed.

According to the requirement of the processing of the screw, $h_{1}$ and $h_{2}$ should change in the range $[-3 ; 3]$ and [-30; 30], respectively; that is, $h_{1}^{d}=-3, h_{1}^{u}=3, h_{2}^{d}=-30$, and $h_{2}^{u}=30$. At the same time, the normal working ranges of the two characteristic variables are $[-2 ; 2]$ and $[-20 ; 20]$. According to (7), the $\mathrm{BRB}$ for determining the running condition of the screw, that is, BRB_3, can be constructed. There are 9 belief rules in BRB_3, shown in Table 5. In addition, $p=6, B_{\text {th }}=0.8$. 
TABLE 6: Updated belief degrees of BRB_1.

\begin{tabular}{lccc}
\hline Rule number & Updated rule weight & $h_{1}(t-1)$ and $h_{2}(t-2)$ & $\left\{D_{1}^{1}, D_{2}^{1}, D_{3}^{1}\right\}$ \\
\hline 1 & 0.9976 & $\mathrm{~S}$ and S & $\left\{\left(D_{1}^{1}, 0.7299\right),\left(D_{2}^{1}, 0.27\right),\left(D_{3}^{1}, 0.0001\right)\right\}$ \\
2 & 0.9677 & $\mathrm{~S}$ and $\mathrm{M}$ & $\left\{\left(D_{1}^{1}, 0.3\right),\left(D_{2}^{1}, 0.7\right),\left(D_{3}^{1}, 0\right)\right\}$ \\
3 & 1 & $\mathrm{~S}$ and $~$ & $\left\{\left(D_{1}^{1}, 1\right),\left(D_{2}^{1}, 0\right),\left(D_{3}^{1}, 0\right)\right\}$ \\
4 & 1 & $\mathrm{M}$ and & $\left\{\left(D_{1}^{1}, 1\right),\left(D_{2}^{1}, 0\right),\left(D_{3}^{1}, 0\right)\right\}$ \\
5 & 1 & $\mathrm{M}$ and M & $\left\{\left(D_{1}^{1}, 0\right),\left(D_{2}^{1}, 1\right),\left(D_{3}^{1}, 0\right)\right\}$ \\
6 & 1 & $\mathrm{M}$ and L & $\left\{\left(D_{1}^{1}, 0\right),\left(D_{2}^{1}, 0.2\right),\left(D_{3}^{1}, 0.8\right)\right\}$ \\
7 & 0.8982 & $\mathrm{~L}$ and S & $\left\{\left(D_{1}^{1}, 1\right),\left(D_{2}^{1}, 0\right),\left(D_{3}^{1}, 0\right)\right\}$ \\
8 & 0.6272 & $\mathrm{~L}$ and M & $\left\{\left(D_{1}^{1}, 0\right),\left(D_{2}^{1}, 0.5\right),\left(D_{3}^{1}, 0.5\right)\right\}$ \\
9 & 1 & $\mathrm{~L}$ and L & $\left\{\left(D_{1}^{1}, 0\right),\left(D_{2}^{1}, 0.0998\right),\left(D_{3}^{1}, 0.0002\right)\right\}$ \\
\hline
\end{tabular}

TABLE 7: Updated belief degrees of BRB_2.

\begin{tabular}{lccc}
\hline Rule number & Updated rule weight & $h_{1}(t-1)$ and $h_{2}(t-2)$ & $\left\{D_{1}^{2}, D_{2}^{2}, D_{3}^{2}\right\}$ \\
\hline 1 & 1 & $\mathrm{~S}$ and S & $\left\{\left(D_{1}^{2}, 0.7499\right),\left(D_{2}^{2}, 0.25\right),\left(D_{3}^{2}, 0.0001\right)\right\}$ \\
2 & 1 & $\mathrm{~S}$ and M & $\left\{\left(D_{1}^{2}, 0.2999\right),\left(D_{2}^{2}, 0.7\right),\left(D_{3}^{2}, 0.0001\right)\right\}$ \\
3 & 1 & $\mathrm{~S}$ and $\mathrm{H}$ & $\left\{\left(D_{1}^{2}, 1\right),\left(D_{2}^{2}, 0\right),\left(D_{3}^{2}, 0\right)\right\}$ \\
4 & 0.9999 & $\mathrm{M}$ and & $\left\{\left(D_{1}^{2}, 1\right),\left(D_{2}^{2}, 0\right),\left(D_{3}^{2}, 0\right)\right\}$ \\
5 & 1 & $\mathrm{M}$ and $\mathrm{M}$ & $\left\{\left(D_{1}^{2}, 0.0998\right),\left(D_{2}^{2}, 0.9\right),\left(D_{3}^{2}, 0.0002\right)\right\}$ \\
6 & 1 & $\mathrm{M}$ and L & $\left\{\left(D_{1}^{2}, 0\right),\left(D_{2}^{2}, 0.2\right),\left(D_{3}^{2}, 0.8\right)\right\}$ \\
7 & 1 & $\mathrm{~L}$ and S & $\left\{\left(D_{1}^{2}, 1\right),\left(D_{2}^{2}, 0\right),\left(D_{3}^{2}, 0\right)\right\}$ \\
8 & 1 & $\mathrm{~L}$ and M & $\left\{\left(D_{1}^{2}, 0\right),\left(D_{2}^{2}, 0.5\right),\left(D_{3}^{2}, 0.5\right)\right\}$ \\
9 & 0.9999 & $\mathrm{~L}$ and L & $\left\{\left(D_{1}^{2}, 0\right),\left(D_{2}^{2}, 0.0998\right),\left(D_{3}^{2}, 0.0002\right)\right\}$ \\
\hline
\end{tabular}

TABLE 8: The MAPE between testing data and prediction values under different model of $h_{1}$.

\begin{tabular}{lcc}
\hline & $\begin{array}{c}\text { Initial forecasting } \\
\text { model }\end{array}$ & $\begin{array}{c}\text { Updated } \\
\text { forecasting model }\end{array}$ \\
\hline MAPE (\%) & 18.85 & 1.49 \\
\hline
\end{tabular}

TABLE 9: The MAPE between testing data and prediction values under different model of $h_{2}$.

\begin{tabular}{lcc}
\hline & $\begin{array}{c}\text { Initial forecasting } \\
\text { model }\end{array}$ & $\begin{array}{c}\text { Updated } \\
\text { forecasting model }\end{array}$ \\
\hline MAPE (\%) & 6.31 & 5.03 \\
\hline
\end{tabular}

According to the initial models and ER approach, the initial prediction models of $h_{1}$ and $h_{2}$ are calculated in Figures 7 and 8 , respectively. It can be seen that the initial prediction values cannot match the testing data very well. It means that the initial prediction models are not accurate. It is necessary to update the parameters by using the effective information. The rules weights and belief rules of update BRBs are listed in Tables 6 and 7. The updated forecasting values generated by the updated prediction models are shown in Figures 7 and 8. From the two figures, it can be seen that the updated models can predict the characteristic variables accurately compared with the initial prediction models. Using the BRB_3, the prediction failure probability, in other words, the forecast results are obtained in Figure 9. Among them, data beyond the threshold show that the system is in a fault condition.

In order to further demonstrate the accuracy of the updated model, mean absolute percentage error (MAPE) is selected as a measure. Tables 8 and 9 list the MAPEs between

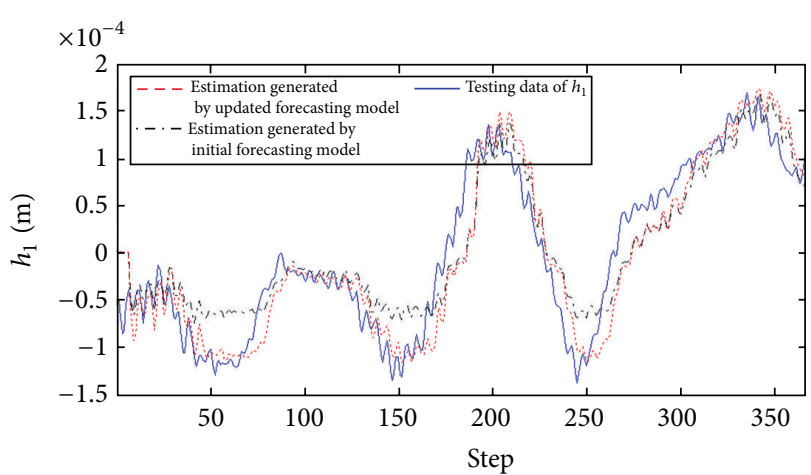

FIGURE 7: Testing data and the estimation generated by the initial and updated model based on BRB of $h_{1}$.

the testing data and the values generated by the initial and the update predicting models of $h_{1}$ and $h_{2}$, respectively. Therefore, the updated models can predict characteristic variables accurately.

The above statement is the process of fault prediction of screw. According to this method, the whole CNC machine tool servo system fault prediction can be finished. Figure 9 shows the result of the screw fault prediction. Through the forecast value, not only failures can be seen, but also the failure process.

\section{Conclusion}

In this paper, the characteristics of the complex electromechanical systems are analyzed deeply. Then the systems model 


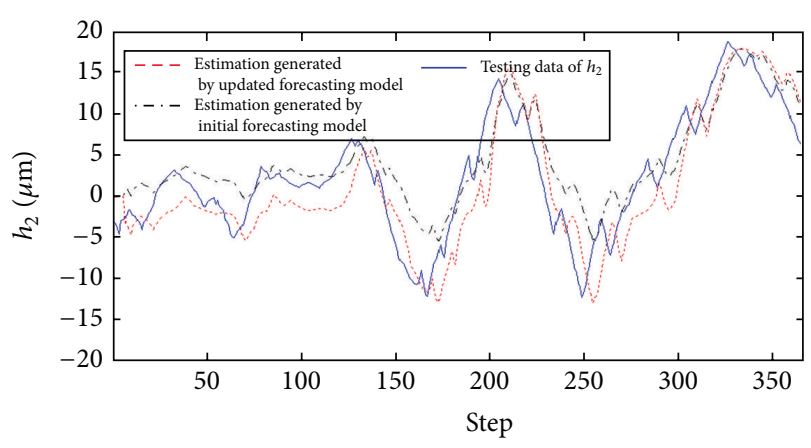

FIGURE 8: Testing data and the estimation generated by the initial and updated model based on BRB of $h_{2}$.

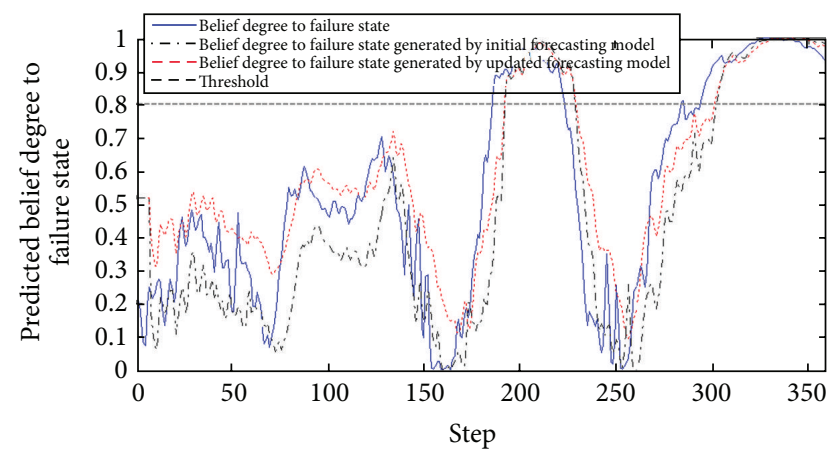

FIGURE 9: Fault prediction results by the initial and updated model based on BRB.

is established. BRB is applied to the fault prediction of complex electromechanical systems. BRB fault prediction model does not need analytical models of the systems that are suitable for the nonlinear characteristics of complex electromechanical system. BRB can effectively make use of prior knowledge and semiquantitative information. Combined with ER algorithm, the fault prediction can meet the real-time requirements of practical engineering. In forecasting results, $\mathrm{BRB}$ can give forecast values of the fault state directly which have the visual interpretation of the results. Characteristic variables prediction models established in this paper are independent of each other. In a practical engineering system, feature variables are not completely independent. In the further study, the algorithm will be improved to change the forecast results more closely to the actual ones.

\section{Conflict of Interests}

The authors declare that there is no conflict of interests regarding the publication of this paper.

\section{Acknowledgments}

This work is supported by the National Nature Science Foundation under Grants 61374138 and 61370031 and the Foundation of the Department of Education of Jilin Province of China under Grant 2009109.

\section{References}

[1] J. Zhong and H. Tang, "Vibration problems of high speed rolling mill-study of dynamics of complex electromechanically coupled system," Journal of Vibration, Measurement and Diagnosis, vol. 22, no. 1, pp. 1-8, 2002.

[2] Y. Zhang, "Review of theory and technology of mechanical reliability for dynamic and gradual systems," Journal of Mechanical Engineering, vol. 49, no. 20, pp. 101-114, 2013.

[3] S.-W. Fei and Y. Sun, "Fault prediction of power transformer by combination of rough sets and grey theory," Proceedings of the Chinese Society of Electrical Engineering, vol. 28, no. 16, pp. 154160, 2008.

[4] J. Zhang, A. J. Morris, and E. B. Martin, "Long-term prediction models based on mixed order locally recurrent neural networks," Computers \& Chemical Engineering, vol. 22, no. 7-8, pp. 1051-1063, 1998.

[5] K. Chakraborty, K. Mehrotra, C. K. Mohan, and S. Ranka, "Forecasting the behavior of multivariate time series using neural networks," Neural Networks, vol. 5, no. 6, pp. 961-970, 1992.

[6] N.Z. Gebraeel and M. A. Lawley, "A neural network degradation model for computing and updating residual life distributions," IEEE Transactions on Automation Science and Engineering, vol. 5, no. 1, pp. 154-163, 2008.

[7] S. L. Ho and M. Xie, "The use of ARIMA models for reliability forecasting and analysis," Computers \& Industrial Engineering, vol. 35, no. 1-2, pp. 213-216, 1998.

[8] M. Chen and D. Zhou, "Fault prediction techniques for dynamic systems," Control Theory \& Applications, vol. 20, no. 6, pp. 819824, 2003.

[9] Z.-J. Zhou, C.-H. Hu, D.-L. Xu, M.-Y. Chen, and D.-H. Zhou, "A model for real-time failure prognosis based on hidden Markov model and belief rule base," European Journal of Operational Research, vol. 207, no. 1, pp. 269-283, 2010.

[10] Z.-J. Zhou, C.-H. Hu, J.-B. Yang, D.-L. Xu, and D.-H. Zhou, "Online updating belief-rule-base using the RIMER approach," IEEE Transactions on Systems, Man, and Cybernetics Part A: Systems and Humans, vol. 41, no. 6, pp. 1225-1243, 2011.

[11] J.-B. Yang, J. Liu, J. Wang, H.-S. Sii, and H.-W. Wang, "Belief rule-base inference methodology using the evidential reasoning approach-RIMER," IEEE Transactions on Systems, Man, and Cybernetics Part A: Systems and Humans, vol. 36, no. 2, pp. 266285, 2006.

[12] J.-B. Yang, J. Liu, D.-L. Xu, J. Wang, and H. Wang, "Optimization models for training belief-rule-based systems," IEEE Transactions on Systems, Man, and Cybernetics Part A: Systems and Humans, vol. 37, no. 4, pp. 569-585, 2007.

[13] D.-L. Xu, J. Liu, J.-B. Yang et al., "Inference and learning methodology of belief-rule-based expert system for pipeline leak detection," Expert Systems with Applications, vol. 32, no. 1, pp. 103-113, 2007.

[14] Z.-J. Zhou, C.-H. Hu, B.-C. Zhang, D.-L. Xu, and Y.-W. Chen, "Hidden behavior prediction of complex systems based on hybrid information," IEEE Transactions on Cybernetics, vol. 43, no. 2, pp. 402-411, 2013.

[15] Z.-J. Zhou, C.-H. Hu, J.-B. Yang, D.-L. Xu, and D.-H. Zhou, "Online updating belief rule based system for pipeline leak detection under expert intervention," Expert Systems with Applications, vol. 36, no. 4, pp. 7700-7709, 2009.

[16] Z.-J. Yang, Q.-B. Hao, F. Chen, B.-B. Xu, X.-B. Li, and H.-W. Zhao, "A comprehensive fuzzy reliability allocation method of 
NC machine tools based on interval analysis," Journal of Beijing University of Technology, vol. 37, no. 3, pp. 321-329, 2011.

[17] J. Hu and L. Zhang, "Opportunistic maintenance strategy for complex oil and gas production equipment based on fault preventive defense," Journal of Mechanical Engineering, vol. 49, no. 12, pp. 167-175, 2013.

[18] Y. Zhang, G. Shen, Y. Jia, D. Xue, and Y. Li, "Research on the disciplinarian and reliability of failure distribution of CNC lathe," Transactions of the Chinese Society of Agricultural Machinery, vol. 37, no. 1, pp. 156-159, 2006.

[19] D. Chen, T. Wang, and H. Wei, "Sectional model involving two Weibull distributions for CNC lathe failure probability," Journal of Beijing University of Aeronautics and Astronautics, vol. 31, no. 7, pp. 766-769, 2005.

[20] Y. Wang, Y. Jia, J. Yu, Y. Zheng, and S. Yi, "Failure probabilistic model of CNC lathes," Reliability Engineering \& System Safety, vol. 65, no. 3, pp. 307-314, 1999.

[21] Z.-J. Zhou, C.-H. Hu, W.-B. Wang, B.-C. Zhang, D.-L. Xu, and J.-F. Zheng, "Condition-based maintenance of dynamic systems using online failure prognosis and belief rule base," Expert Systems with Applications, vol. 39, no. 6, pp. 6140-6149, 2012. 


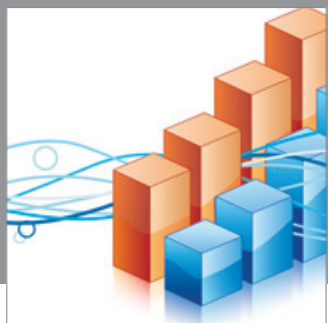

Advances in

Operations Research

mansans

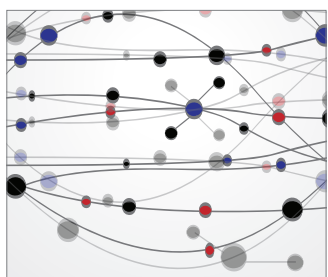

The Scientific World Journal
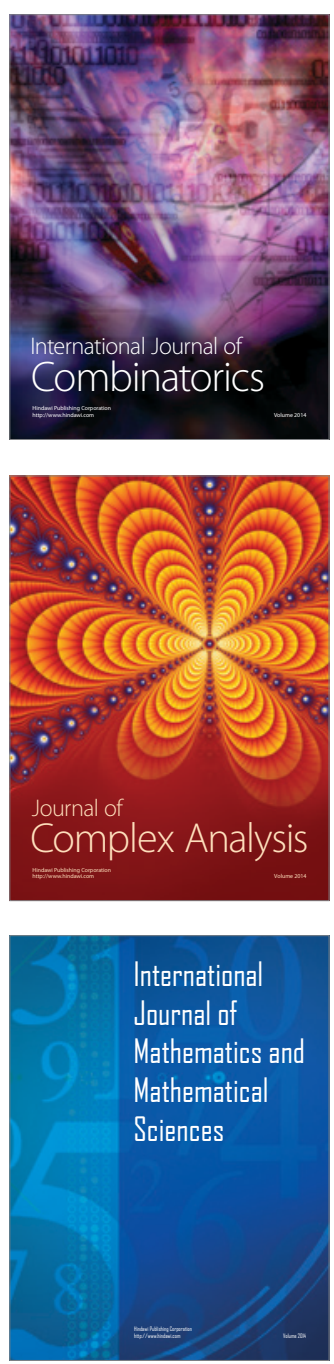
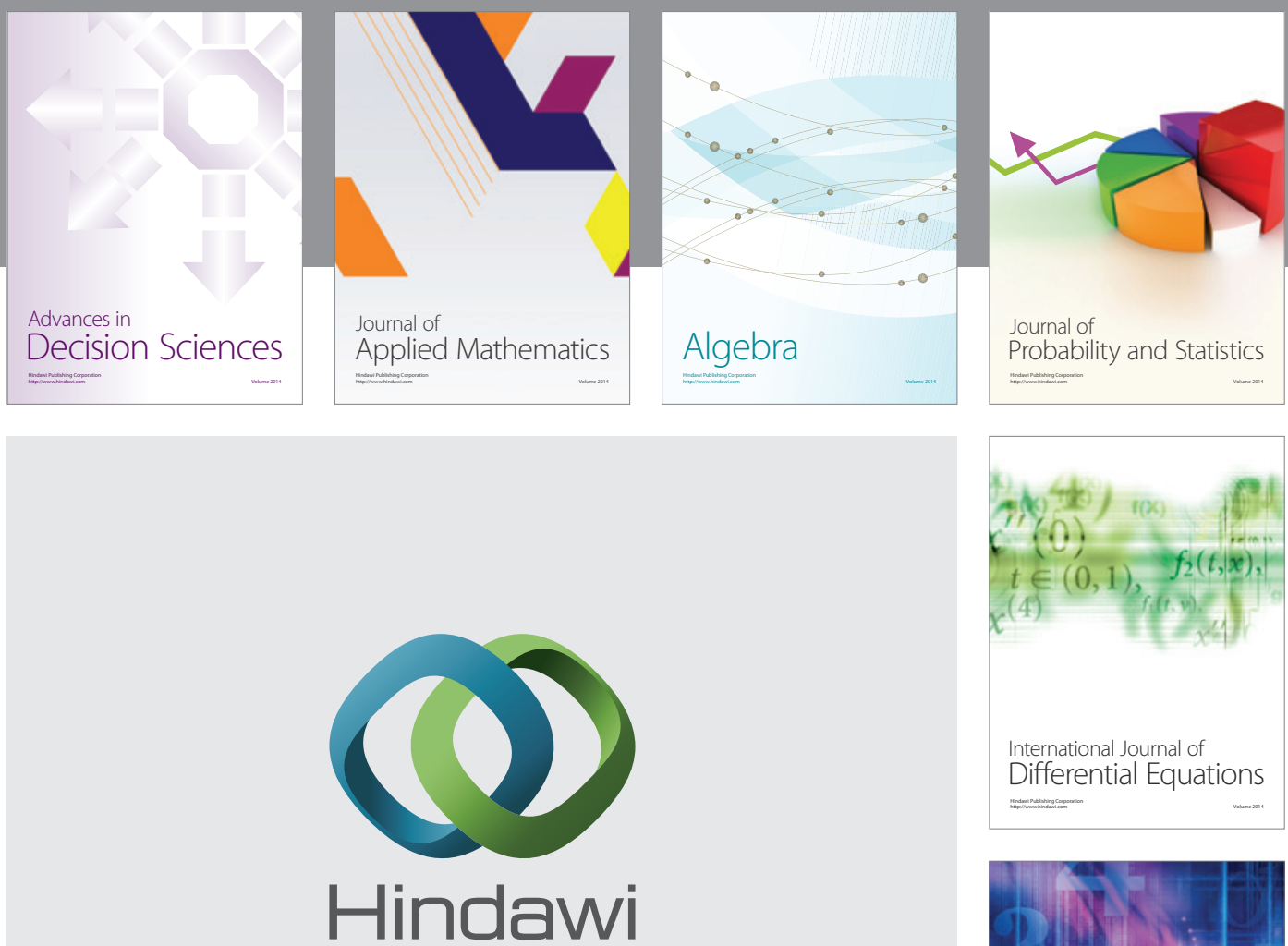

Submit your manuscripts at http://www.hindawi.com
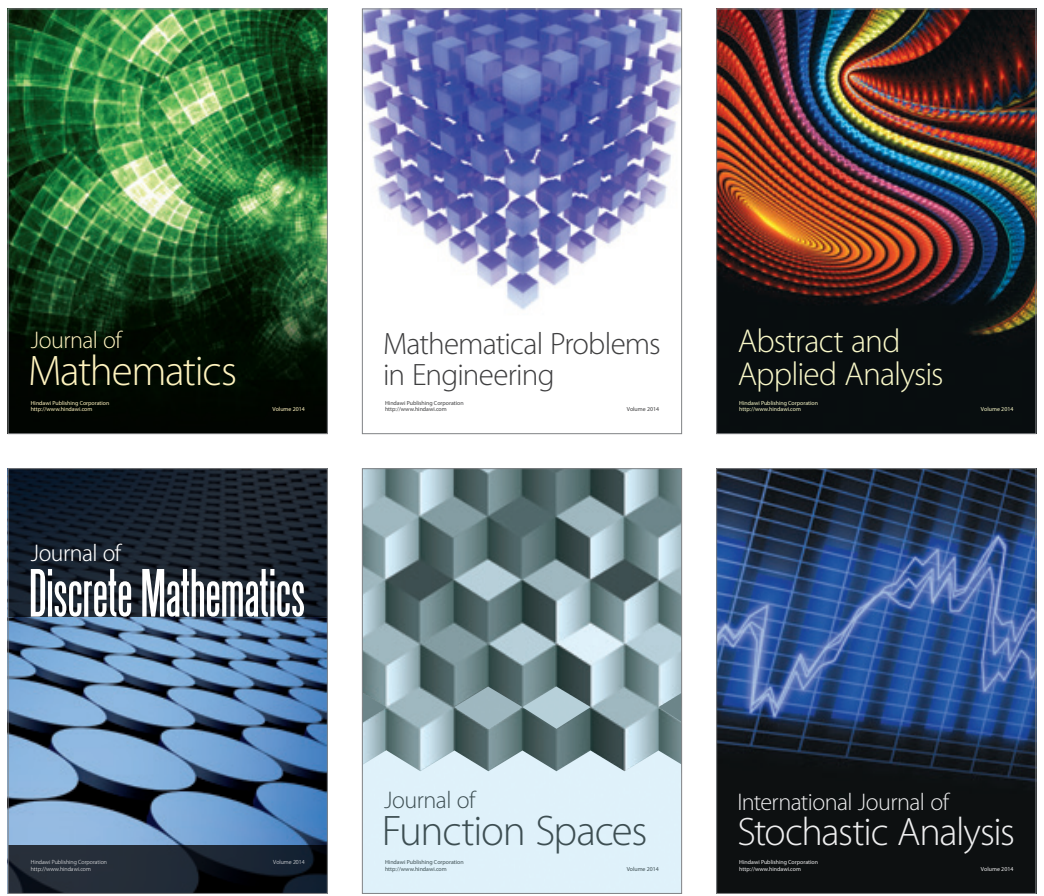

Journal of

Function Spaces

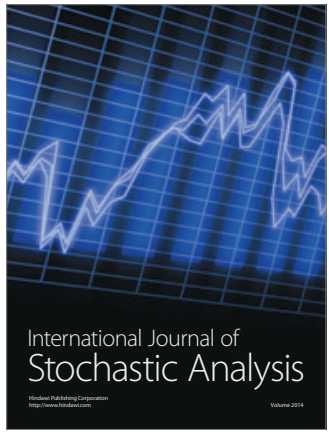

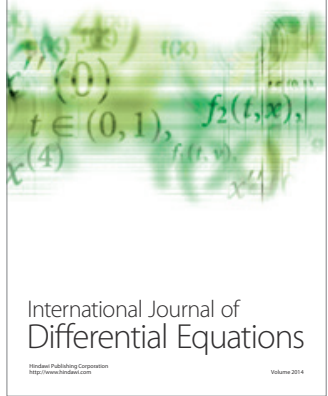
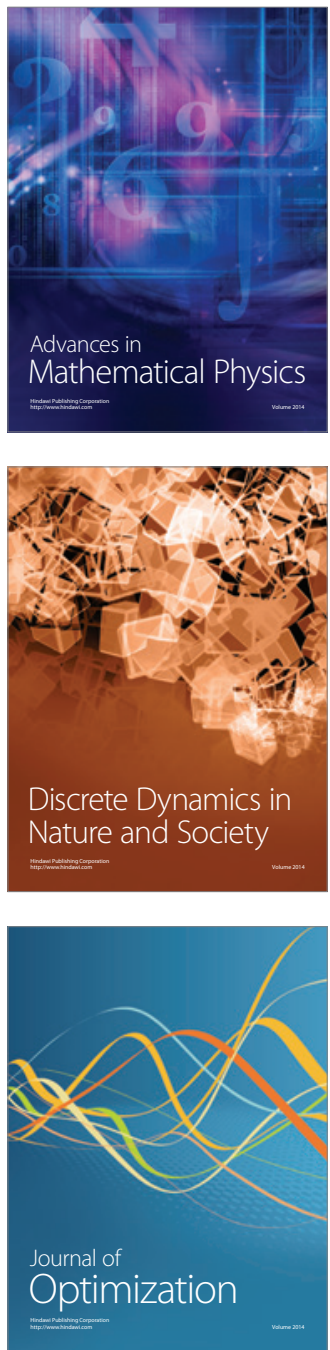